\title{
Protein encoding genes in an ancient plant: analysis of codon usage, retained genes and splice sites in a moss, Physcomitrella patens Stefan A Rensing*, Dana Fritzowsky, Daniel Lang and Ralf Reski
}

\author{
Address: Plant Biotechnology, Faculty of Biology, University of Freiburg, Schaenzlestr. 1, 79104 Freiburg, Germany \\ Email: Stefan A Rensing* - stefan.rensing@biologie.uni-freiburg.de; Dana Fritzowsky - dana@fritzowsky.net; \\ Daniel Lang - daniel.lang@biologie.uni-freiburg.de; Ralf Reski - ralf.reski@biologie.uni-freiburg.de \\ * Corresponding author
}

Published: 22 March 2005

BMC Genomics 2005, 6:43 doi:10.1 |86/|47|-2/64-6-43
Received: 24 September 2004

Accepted: 22 March 2005

This article is available from: http://www.biomedcentral.com/I47I-2/64/6/43

(C) 2005 Rensing et al; licensee BioMed Central Ltd.

This is an Open Access article distributed under the terms of the Creative Commons Attribution License (http://creativecommons.org/licenses/by/2.0), which permits unrestricted use, distribution, and reproduction in any medium, provided the original work is properly cited.

\begin{abstract}
Background: The moss Physcomitrella patens is an emerging plant model system due to its high rate of homologous recombination, haploidy, simple body plan, physiological properties as well as phylogenetic position. Available EST data was clustered and assembled, and provided the basis for a genome-wide analysis of protein encoding genes.
\end{abstract}

Results: We have clustered and assembled Physcomitrella patens EST and CDS data in order to represent the transcriptome of this non-seed plant. Clustering of the publicly available data and subsequent prediction resulted in a total of $19,08 \mathrm{I}$ non-redundant ORF. Of these putative transcripts, approximately $30 \%$ have a homolog in both rice and Arabidopsis transcriptome.

More than 130 transcripts are not present in seed plants but can be found in other kingdoms. These potential "retained genes" might have been lost during seed plant evolution. Functional annotation of these genes reveals unequal distribution among taxonomic groups and intriguing putative functions such as cytotoxicity and nucleic acid repair.

Whereas introns in the moss are larger on average than in the seed plant Arabidopsis thaliana, position and amount of introns are approximately the same. Contrary to Arabidopsis, where CDS contain on average $44 \%$ G/C, in Physcomitrella the average G/C content is $50 \%$. Interestingly, moss orthologs of Arabidopsis genes show a significant drift of codon fraction usage, towards the seed plant. While averaged codon bias is the same in Physcomitrella and Arabidopsis, the distribution pattern is different, with 15\% of moss genes being unbiased.

Species-specific, sensitive and selective splice site prediction for Physcomitrella has been developed using a dataset of 368 donor and acceptor sites, utilizing a support vector machine. The prediction accuracy is better than those achieved with tools trained on Arabidopsis data.

Conclusion: Analysis of the moss transcriptome displays differences in gene structure, codon and splice site usage in comparison with the seed plant Arabidopsis. Putative retained genes exhibit possible functions that might explain the peculiar physiological properties of mosses.

Both the transcriptome representation (including a BLAST and retrieval service) and splice site prediction have been made available on http://www.cosmoss.org, setting the basis for assembly and annotation of the Physcomitrella genome, of which draft shotgun sequences will become available in 2005. 


\section{Background}

Flowering plants have developed from a common ancestor with mosses, liverworts, ferns, and gymnosperms over the last 450 million years [1]. Most recent angiosperms do not closely resemble their ancestors, as known from the fossil record. Quite a few gymnosperms (like Ginkgo or Cycas) still resemble the plants known from the fossil record, and this is even more true for "lower" land plants, namely mosses, liverworts and ferns [2,3]. In addition, mosses seem to evolve with a slow molecular clock [4]. So, if these plants appear to be more ancient than modern flowering plants as measured by morphological means and mutation rate, does this also hold true for how they employ their genetic system?

A lot of comparative studies on protein encoding genes have already been carried out within and between the two major groups of flowering plants, mono- and dicotyledons, with rice (Oryza sativa) and Arabidopsis thaliana as the most prominent examples. Currently more than two million Liliopsida (monocotyledons) EST are publicly available, the corresponding number for the Magnoliophyta (dicotyledons) even exceeds four million sequences. However, sequence information for other plant phyla is still scarce. There are only about 160,000 EST sequences available of both Coniferophyta (part of the gymnosperms) and Chlorophyta (green algae), 130,000 from Bryophyta (mosses) and 3,700 from Filicophyta (ferns) (all numbers from Genbank). For the moss Physcomitrella patens, more than 102,000 nucleic acid sequences (mainly EST) are publicly available to date. This "ancient" land plant therefore is an ideal candidate to unravel some details about how simple plants encode proteins and whether they do so in a different manner from "modern" plants, as represented by the monocotyledon rice and the dicotyledon Arabidopsis in this study.

Physcomitrella is increasingly being used as a model plant because of its unrivalled capability among plants to include ectopic DNA into its genome by means of homologous recombination (see e.g. [5-7]), thus enabling gene replacement in a straight forward manner. As in all mosses, the haploid gametophyte is the dominant generation in the heteromorphic life cycle. In this respect the moss is different from seed plants (gymnosperms and flowering plants), in which the polyploid sporophyte dominates the life cycle. It has been argued before $[8,9]$ that the set of genes of the respective dominant generation is equivalent, while a large proportion of moss transcripts cannot currently be assigned a putative function. These "orphan" genes might encode functions that are specific to mosses and are not present in other taxonomic groups. Besides species-specific orphan genes, mosses might also possess retained genes, that have been lost in seed plants during evolution. Both types of genes are candidates to

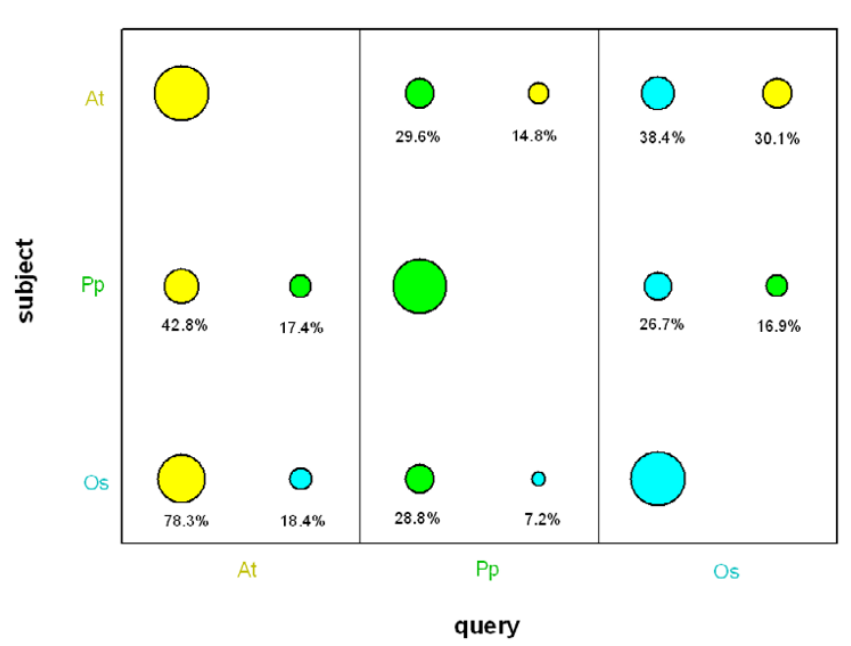

\section{Figure I}

Comparative BLAST searches between Arabidopsis, rice and moss. Comparative BLAST searches of the Arabidopsis (At, yellow), rice (Os, cyan) and Physcomitrella ( $\mathrm{Pp}$, green) transcriptomes. Each search was done with the respective sets once as query and once as search space (subject). The area of the circles represents the percentage of the query/subject sequence space that yielded filtered hits.

encode functions that make mosses unique in terms of physiology and metabolism. For example, Physcomitrella exhibits increased tolerance towards abiotic stresses $[10,11]$, uses proteins derived from the same gene in different cellular compartments by dual targeting $[12,13]$ and displays secondary metabolite pathways not known in seed plants [14-16]. In this study, following up on the initial analyses by Nishiyama et al. [8], we aimed to increase our knowledge of the moss transcriptome.

\section{Results and discussion Comparative BLAST searches}

Around $30 \%$ of the Physcomitrella ORF have homologs in both rice and Arabidopsis transcriptome whereas $80 \%$ of the Arabidopsis genes have a homolog in rice and $40 \%$ of the rice genes in Arabidopsis (Fig. 1). Although these numbers are lower than the actual amount of sequence homologs because of filtering (see below), they demonstrate that Physcomitrella contains a lot of as yet unknown protein encoding genes that might be specific for mosses. A homology search against the taxprot dataset (Table 1, Fig. 2) reveals that $45.8 \%$ of the predicted moss ORF find a query in plants (E-value threshold 1E-4), after rigorous filtering $28.1 \%$ remain and $21.7 \%$ are non-redundant, i.e. do not match multiple subject sequences. The rigorous filtering (see methods for details) for true homologs thus 
Table I: Taxonomic constitution of the taxprot dataset

\begin{tabular}{lrr}
\hline taxonomic group & txid & $\begin{array}{r}\text { \# of } \\
\text { sequences }\end{array}$ \\
\hline Metazoa & 33208 & 862,420 \\
Fungi & 4751 & 184,282 \\
Viridiplantae (plants and green algae) & 33090 & 293,156 \\
Non-green algae ${ }^{2}$ & & 21,889 \\
Other Eukaryotes & & 49,732 \\
Eubacteria (without Cyanobacteria) & 2 & $1,386,089$ \\
Cyanobacteria & 1117 & 94,920 \\
Archaea & 2157 & 122,394 \\
Viruses & 10239 & 331,246 \\
Total & & $\mathbf{3 , 3 4 6 , 1 2 8}$ \\
\hline
\end{tabular}

'Genbank amino acid sequences as of 2004-04-07, NCBI taxon ids are shown under "txid", all taxonomic crown groups with at least 100 sequence members were used; ${ }^{2}$ Cercozoa [136419], Cryptophyta [3027], Euglenozoa [33682], Glaucocystophyceae [38254],

Haptophyceae [2830], Rhodophyta [2763], Stramenopiles [33634];

${ }^{3}$ Acanthamoebidae [33677], Alveolata [33630], Diplomonadida

[207245], Entamoebidae [33084], Heterolobosea [5752], Jakobidae

[143015], Mycetozoa [142796], Parabasalidea [5719]

necessarily decreases the set of available sequences, so that false conclusions are not made based on comparison of non-homologous sequences.

\section{Full-length transcripts}

The total number of clusters after EST clustering do not equal the number of protein encoding genes. This is mainly due to partial (as opposed to full-length) transcripts, i.e. a single gene is represented by more than one sequence because they do not overlap. How many of the clustered public (PPP) transcripts represent full-length coding sequences? Of those sequences that yield a filtered hit against plant mRNAs, $7.9 \%$ are putatively full-length. Of those, $53.9 \%$ start with Methionine, of the latter, $32.4 \%$ contain no X (X represents an indeterminable codon, which can be included by the ORF prediction).

\section{Orthologs, paralogs and mapping}

The filtered hits against the Arabidopsis transcriptome $(1,994$ in total) were divided into non-redundant orthologs (722) and paralogs $(1,015)$. As Arabidopsis orthologs, we defined all those sequences for which the initial subject matches the query in the reverse search (reciprocal hit). Paralogs were defined as those sequences for which this rule does not apply. This method of detecting potential orthologs has been used previously for crossspecies comparisons (e.g. $[17,18])$. The three sequence sets were mapped against the Arabidopsis chromosomes using BLAST (Fig. 3). The distribution pattern clearly reveals the centromeric regions but otherwise does not display significant differences. Although there are some chromosome and sequence set-specific differences in the rate of hits per Mbp, these are not significant as measured by absolute average deviation.

\section{Taxonomic distribution and retained genes}

The highest total number of non-redundant, filtered BLAST hits is (as expected) derived from the plant subset of the taxprot dataset (Table 1, Fig. 2a), followed by the animal and fungi subsets. When looking at the hits as percentage of the search space size (Fig. 2b), it becomes evident that quite a proportion of the sequence space of lower eukaryotes (including non-green algae) is covered. The comparatively high coverage of this "ancient" gene space suggests that mosses share many specialized genes with unicellular organisms.

134 Physcomitrella ORF have their best BLAST hit not among plants (Fig. 4a). Consequently, these are candidates for horizontal gene transfer or, more likely, retained genes that were lost in seed plants during evolution. We had a closer look at those 57 transcripts which are specific to a single taxonomic group, namely bacteria, cyanobacteria, animals or fungi (unique hits). For 25 of those, a putative function could be assigned manually (Fig. 4b, Table 2 ). The broad functional categories of these taxon-specific retained genes are to some extent unevenly distributed. Whereas transport associated proteins are found solely among fungi, signal transduction gene products are found in both bacteria and animals. Transport and metabolism associated gene products support the wealth of secondary pathways found in moss (e.g., [14,15,19-22]), whereas the signal transduction genes also separate the moss from seed plants in this regard. Of special interest are two other functional categories among these candidate retained genes: cytotoxicity and nucleic acid modification. A broad range of cytotoxic abilities might explain why mosses can survive in moist environments mainly unplagued by microbial parasites, without the protection of a cuticula. Furthermore, it is, up until now, puzzling why Physcomitrella is able to integrate ectopic DNA into the genome by homologous recombination with an extraordinarily high rate $[23,24]$ so far only found in bacteria and yeast, but in no other plant or any animal. Hints to unravel this mystery might be found in the presence of genes involved in DNA repair, binding and modification, as we discovered during this research.

\section{Gene structure and splice sites}

The average rate of introns per gene $(\sim 5)$ is the same in Physcomitrella, Arabidopsis and human [25]. The average Physcomitrella intron (252 bp) is longer than those of Arabidopsis (146 bp) and shorter than the typical human intron (740 bp). Furthermore, the Physcomitrella intron is longer than the exon, whereas in Arabidopsis it is the other way round. While the size distribution of Arabidopsis introns is centered around $70 \mathrm{bp}$, the longer moss introns 
a)

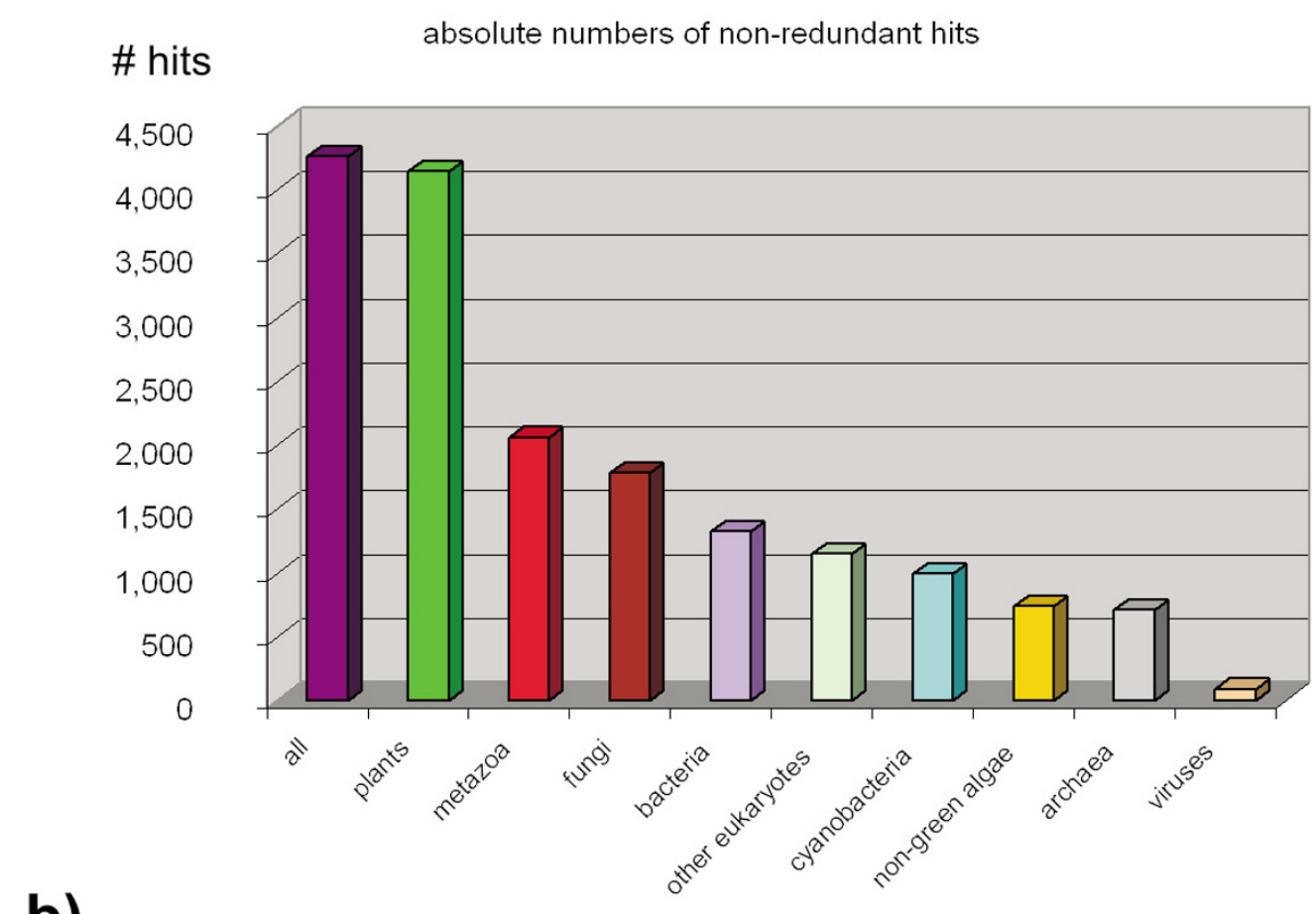

b)

$\%$ hits non redundant hits as $\%$ of sequence space

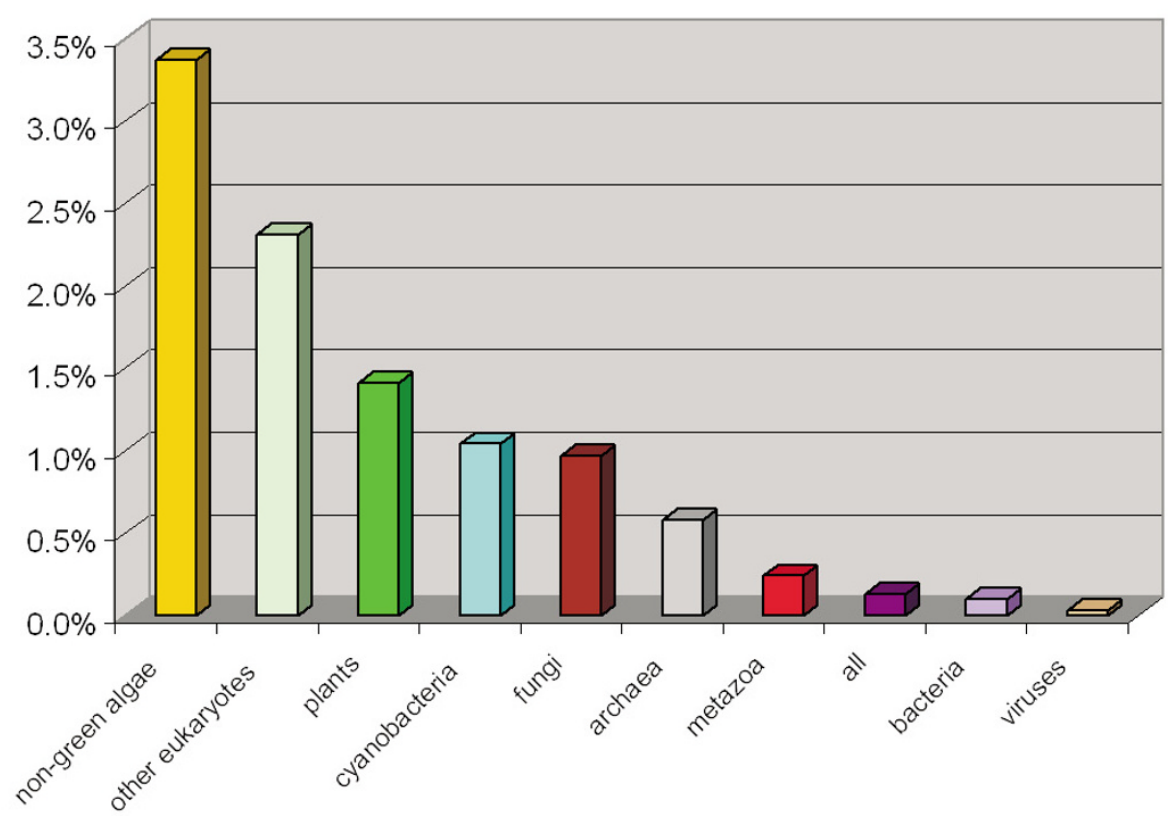

Figure 2

BLAST hits of Physcomitrella protein genes against the taxprot dataset. a) Absolute number of hits against different taxonomic groups. b) Amount of non-redundant hits as percentage of the respective sequence space. 


\section{a)}

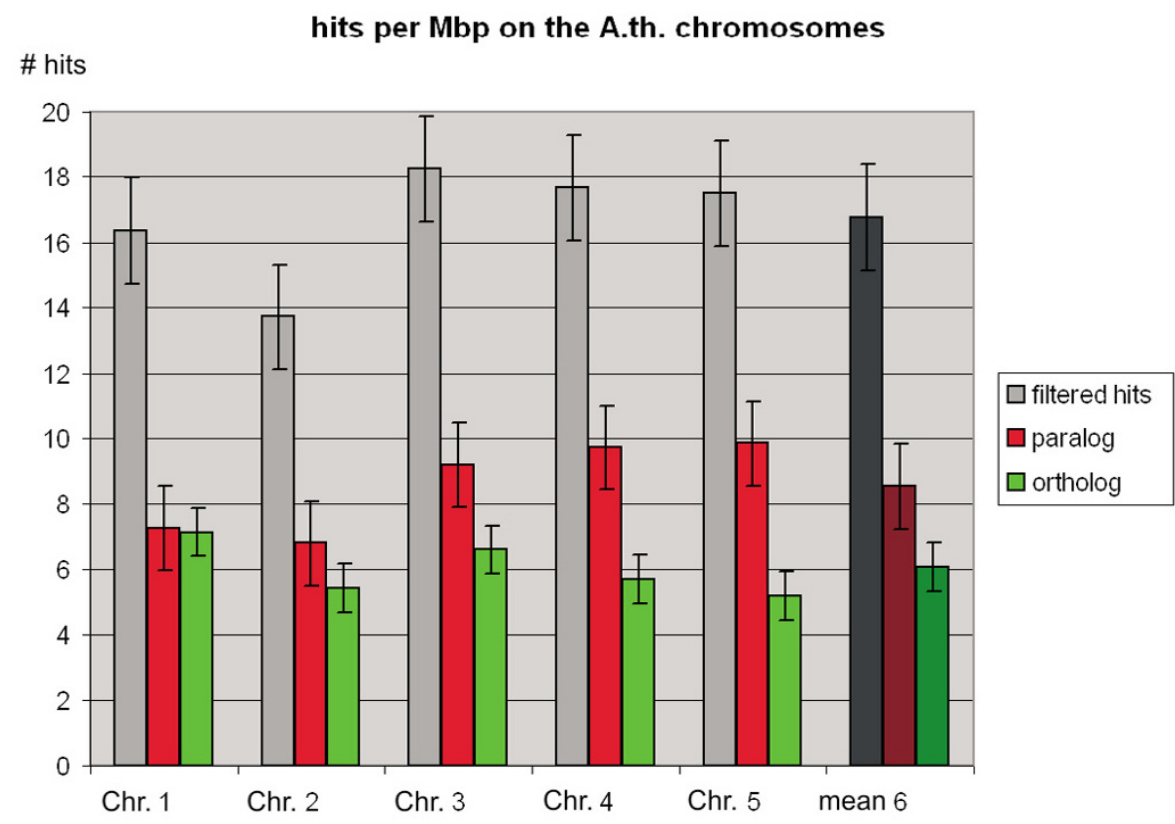

b)

mapping of hits against the A.th. chromosomes

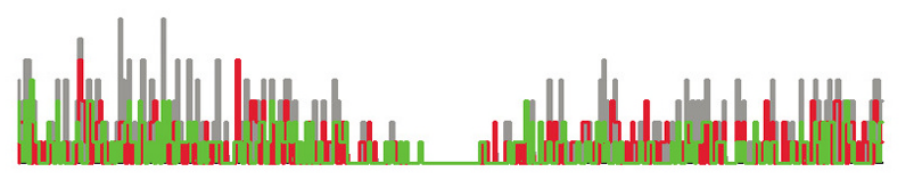

Chr. 1

30Mbp

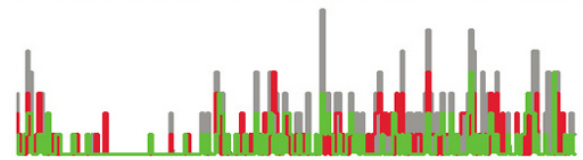

Chr. 2


23Mbp

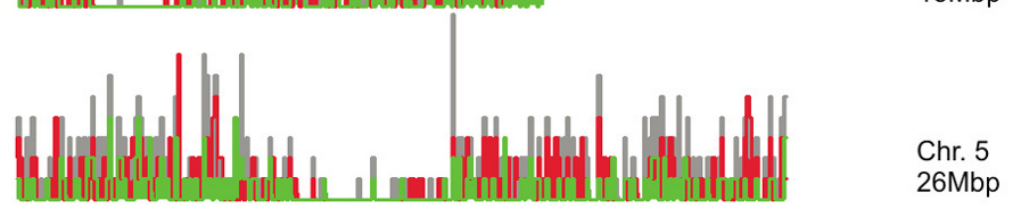

\section{Figure 3}

Mapping of Physcomitrella transcripts to the Arabidopsis chromosomes. Mapping of filtered BLAST hits (grey), paralogs (red) and orthologs (green) against the five Arabidopsis chromosomes (left to right / top to bottom). a) Hits per Mbp; error bars: average absolute deviation (AAD); column 6: mean values. b) Graphical representation using a finer granularity (I00 kbp), each vertical step represents one hit. 
a)

\section{unique hits and functional annotation \\ \# hits}

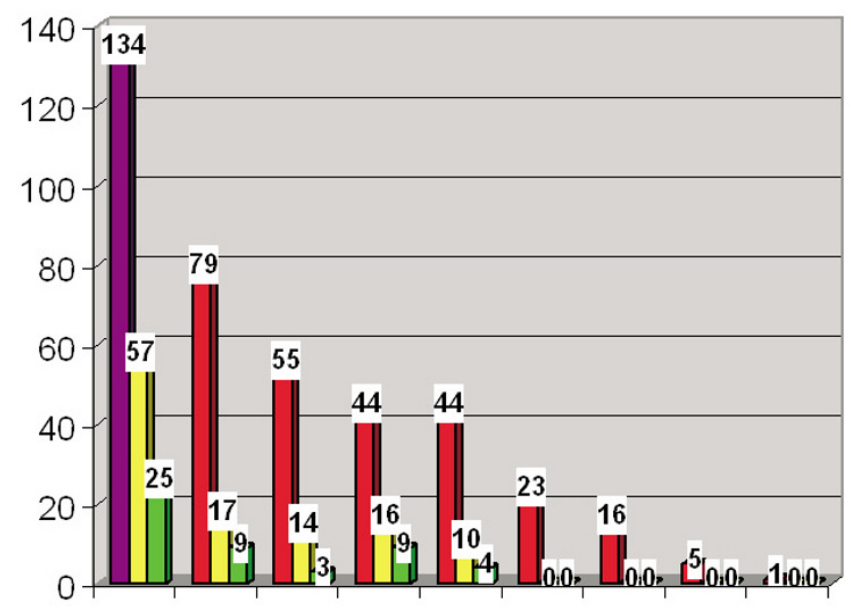

amount of homologs

$\square$ unique (specific) hits

$\square$ functional annotation

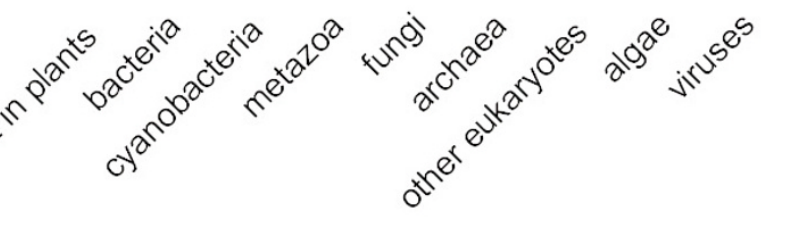

b)

distribution of functional categories

\# seq.

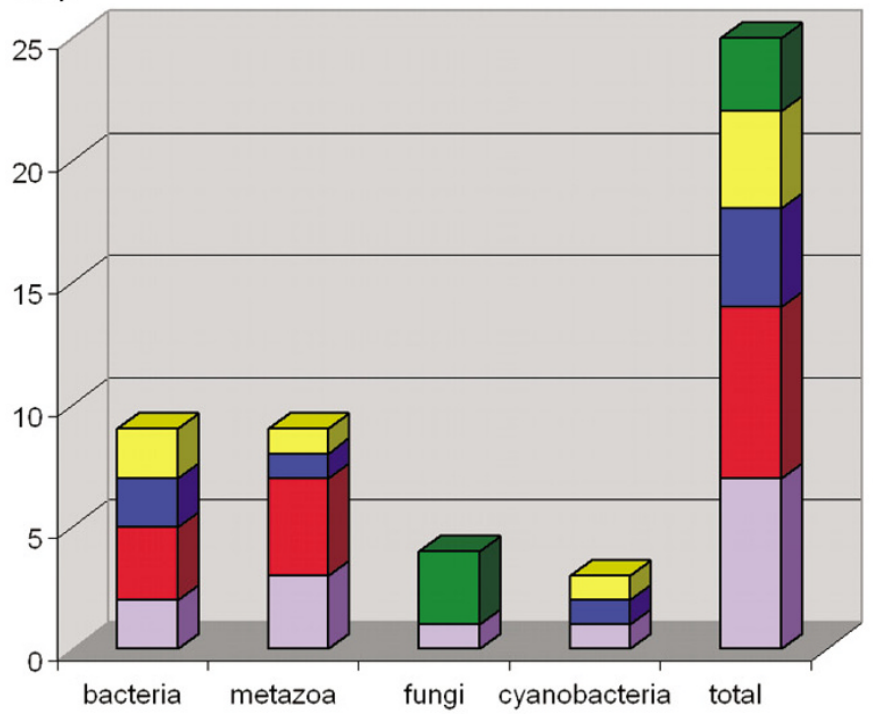

$\square$ transport

$\square$ cytotoxicity

$\square$ n.a. binding / modification

$\square$ signal tansduction

$\square$ metabolism

\section{Figure 4}

Retained genes in moss: taxonomic distribution and functional categories. a) Physcomitrella transcripts which have their best BLAST hit not among plants, divided by taxonomic category, further subdivided into specific hits (unique to a single taxonomic group - yellow) and those that could be assigned a putative function by means of homology searches (green). b) Distribution of functional categories among those taxonomic groups that yielded unique hits. 
Table 2: Functional annotation of retained genes into broad functional categories, assembled transcripts can be retrieved via http:// www.cosmoss.org.

\begin{tabular}{|c|c|c|c|c|c|}
\hline $\begin{array}{l}\text { Pp transcript } \\
\text { (putative } \\
\text { retained gene) }\end{array}$ & $\begin{array}{l}\text { taxonomic } \\
\text { group }\end{array}$ & homolog & $\begin{array}{l}\text { broad functional } \\
\text { category } \\
\text { (potential) }\end{array}$ & $\begin{array}{l}\text { functional } \\
\text { category }\end{array}$ & details \\
\hline PPP_2925_CI & bacteria & $\begin{array}{l}\text { Membrane-bound lytic } \\
\text { murein transglycosylase B }\end{array}$ & cytotoxicity & murein degradation & $\begin{array}{l}\text { murein-degrading enzyme, } \\
\text { may play a role in recycling of } \\
\text { muropeptides during cell }\end{array}$ \\
\hline BJ203770 & bacteria & putative protease & cytotoxicity & protease & \\
\hline PPP_4234_CI & metazoa & cytolysin I & cytotoxicity & cytotoxicity & involved in pore-formation \\
\hline PPP_35I0_Cl & cyano & $\begin{array}{l}\text { RTX toxins and related } \\
\text { Ca2+-binding proteins }\end{array}$ & cytotoxicity & cytotoxicity & \\
\hline PPP_II72_Cl & bacteria & $\begin{array}{l}\text { Enoyl-CoA hydratase/ } \\
\text { carnithine racemase }\end{array}$ & metabolism & fatty acid metabolism & \\
\hline PPP_6629_CI & bacteria & mannosylglycerate synthase & metabolism & sugar metabolism & \\
\hline PPP_5746_Cl & metazoa & $\begin{array}{l}\text { L-kynurenine 3- } \\
\text { monooxygenase Fpk }\end{array}$ & metabolism & $\begin{array}{l}\text { amino acid } \\
\text { metabolism }\end{array}$ & \\
\hline PPP_8479_CI & metazoa & COMMD2 & metabolism & copper metabolism & $\begin{array}{l}\text { COMM (copper metabolism } \\
\text { MURRI) domain containing } 2\end{array}$ \\
\hline BJ 173412 & metazoa & ubiquitin & metabolism & protein metabolism & $\begin{array}{l}\text { ribosomal protein in } \mathrm{C} \text {. } \\
\text { elegans dehydrogenases }\end{array}$ \\
\hline PPP_3987_CI & fungi & MNN9 & metabolism & $\mathrm{N}$-glycosylation & \\
\hline PPP_65I4_CI & cyano & oxidoreductase & metabolism & energy metabolism & $\begin{array}{l}\text { related to aryl-alcohol } \\
\text { dehydrogenases }\end{array}$ \\
\hline PPP_II394_Cl & bacteria & $\begin{array}{c}\text { homolog of eukaryotic DNA } \\
\text { ligase III }\end{array}$ & $\begin{array}{l}\text { nucleic acid binding / } \\
\text { modification }\end{array}$ & DNA repair & \\
\hline BJ 191550 & bacteria & $\begin{array}{l}\text { formamidopyrimidine-DNA } \\
\text { glycosylase }\end{array}$ & $\begin{array}{l}\text { nucleic acid binding / } \\
\text { modification }\end{array}$ & DNA repair & \\
\hline BJ 160862 & metazoa & Osal nuclear protein & $\begin{array}{l}\text { nucleic acid binding / } \\
\text { modification }\end{array}$ & DNA binding & chromatin regulation \\
\hline BJ582496 & cyano & $\begin{array}{l}\text { SAM-dependent } \\
\text { methyltransferase }\end{array}$ & $\begin{array}{l}\text { nucleic acid binding / } \\
\text { modification }\end{array}$ & $\begin{array}{l}\text { nucleic acid } \\
\text { modification }\end{array}$ & \\
\hline PPP_2586_CI & bacteria & CarD protein & signal transduction & DNA binding & $\begin{array}{l}\text { leucine zipper transcription } \\
\text { factor, light- and starvation- } \\
\text { induced response }\end{array}$ \\
\hline PPP_3689_Cl & bacteria & $\begin{array}{l}\text { serine/threonine protein } \\
\text { kinase }\end{array}$ & signal transduction & signal transduction & \\
\hline BJ 172132 & bacteria & $\begin{array}{l}\text { serine/threonine protein } \\
\text { kinase }\end{array}$ & signal transduction & signal transduction & \\
\hline PPP_460_Cl & metazoa & HLA-B-associated transcript & signal transduction & signal transduction & \\
\hline PPP_I04I_CI & metazoa & $\begin{array}{l}\text { calcium/calmodulin- } \\
\text { dependent protein kinase II } \\
\text { delta }\end{array}$ & signal transduction & signal transduction & \\
\hline PPP_6326_Cl & metazoa & tumor suppressor tout-velu & signal transduction & signal transduction & $\begin{array}{l}\text { involved in diffusion of } \\
\text { hedgehog }\end{array}$ \\
\hline PPP_II399_CI & metazoa & $\begin{array}{l}\text { dual-specificity tyrosine } \\
\text { phosphatase YVHI }\end{array}$ & signal transduction & signal transduction & $\begin{array}{l}\text { Non-receptor class dual } \\
\text { specificity subfamily }\end{array}$ \\
\hline PPP_184_CI & fungi & high-affinity iron permease & transport & transport & high affinity iron uptake \\
\hline PPP_7II5_C2 & fungi & uric acid-xanthine permease & transport & transport & $\begin{array}{c}\text { belongs to the Xanthine/Uracil } \\
\text { oermeases family }\end{array}$ \\
\hline PPP_III9I_CI & fungi & $\begin{array}{l}\text { inorganic phosphate } \\
\text { transporter }\end{array}$ & transport & transport & $\begin{array}{c}\text { probable inorganic phosphate } \\
\text { transporter; yeast pho99 } \\
\text { homologue }\end{array}$ \\
\hline
\end{tabular}

are mainly clustered around 180 bp (data not shown). This fits the weak correlation of intron length and genome size generally found in eukaryotic organisms [26]. Intron positions of close homologs between Physcomitrella and Arabidopsis are generally conserved (e.g., [27]).
The Physcomitrella G/C content of $40 \%$ in the intron and $50 \%$ in the exon differs significantly from that of Arabidopsis; $33 \%$ and $44 \%$, respectively. Generally, Physcomitrella introns contain more thymine $(\mathrm{T})$ than the exons. In terms of mononucleotide composition, $\mathrm{T}$ is 
a)


b)
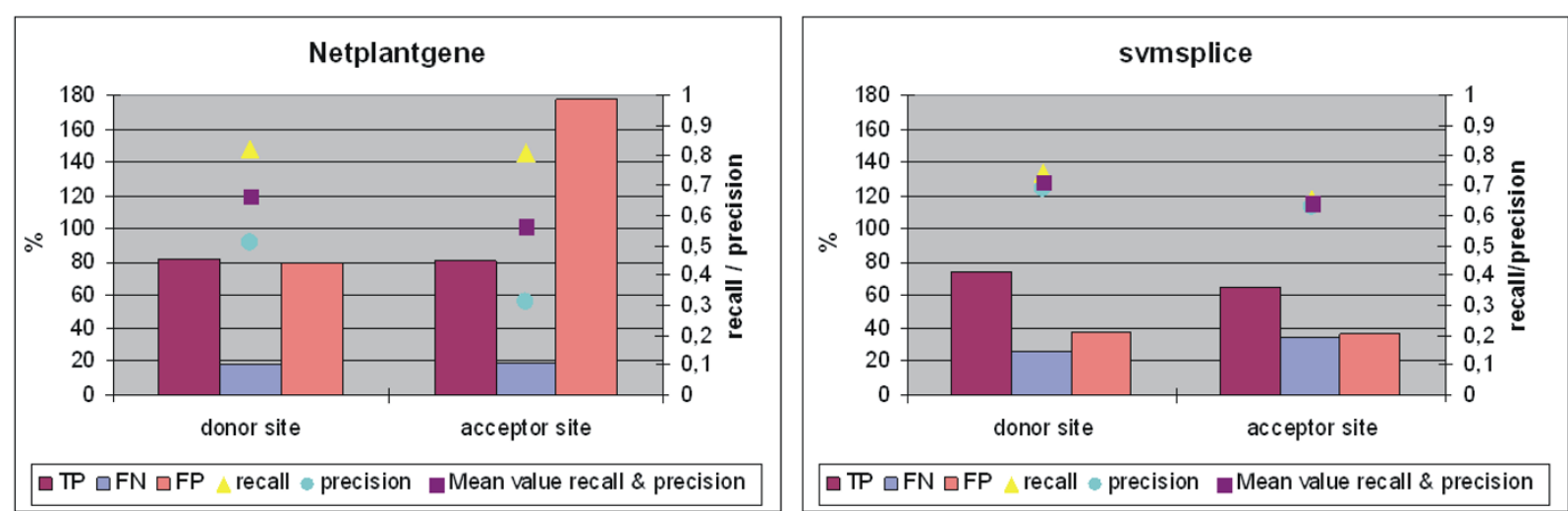

Figure 5

Splice site sequence logos and efficiency of splice site prediction. a) Sequence logos of Physcomitrella donor and acceptor sites. b) Prediction performance of Netplantgene and svmsplice for Physcomitrella splice sites. TP = true positive, FN = false negative, $F P=$ false positive, measured on the lefthand $(\%)$ axis. Recall $($ sensitivity $)=t p /(t p+f n)$, precision $=t p /(t p+f p)$, measured on the righthand axis.

overrepresented in the intron and $\mathrm{C}$ is underrepresented in the exon. In terms of dinucleotides, there is a significant overrepresentation of TT in the introns. Outstanding trinucleotide usage are the overrepresented TTT in the intron and the stop codon TGA in the exon, while the other two stop codons TAA and TAG are underrepresented in the moss.

A visualisation of the Physcomitrella donor and acceptor sites is shown in figure 5a. Comparison of the Arabidopsistrained Netplantgene [25] and the Physcomitrella-trained svmsplice (Fig. 5b) reveals a better overall performance of the support vector machine. Although Netplantgene exhibits a high recall, precision is low, which is due to the large amount of false positive predictions. Svmsplice predicts a lower rate of true positives (thus lower recall), however, precision is much better. The mean values of recall and precision for both donor and acceptor site are higher for svmsplice and thus make it the method of choice for accurate prediction of Physcomitrella splice sites.

\section{Composition of coding sequences and codon usage}

Significant differences in codon fraction usage for the three above mentioned sequence subsets (Arabidopsis orthologs and paralogs, retained genes) when compared with the averaged codon usage in Physcomitrella and Arabidopsis are shown in Fig. 6a. The Average G/C content of the Arabidopsis CDS is 43\%, whereas it is $50 \%$ for Phys- 
a)

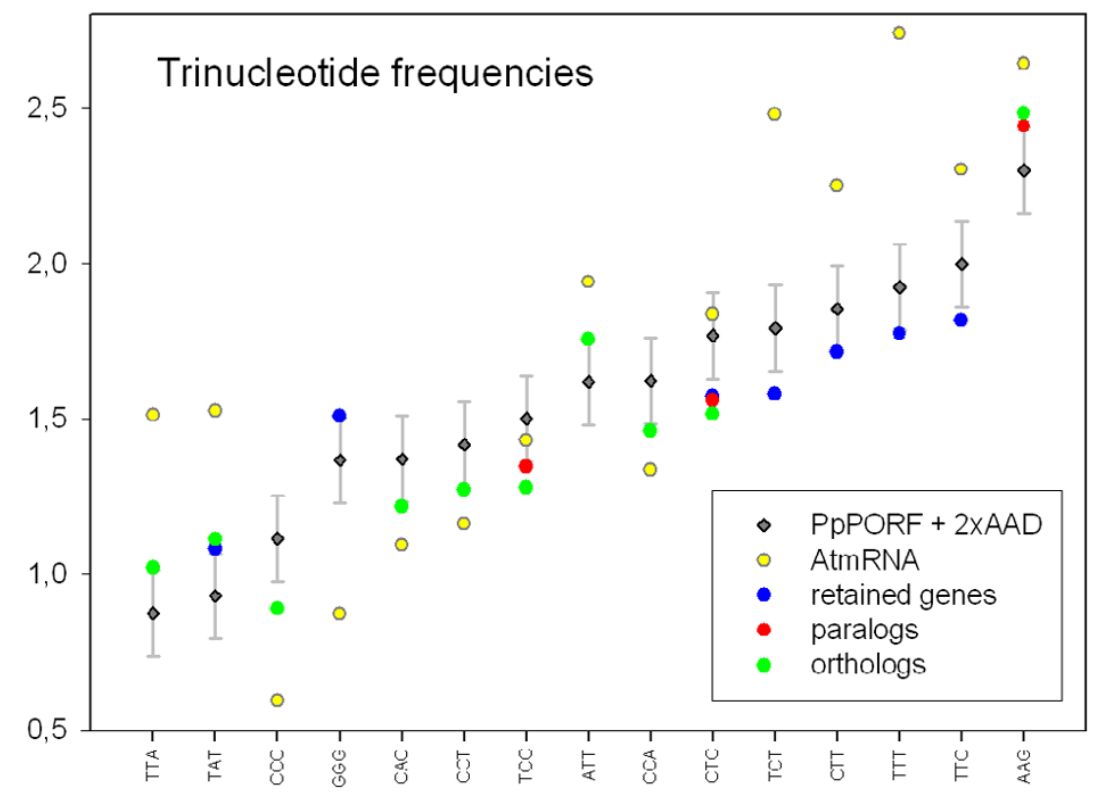

b)

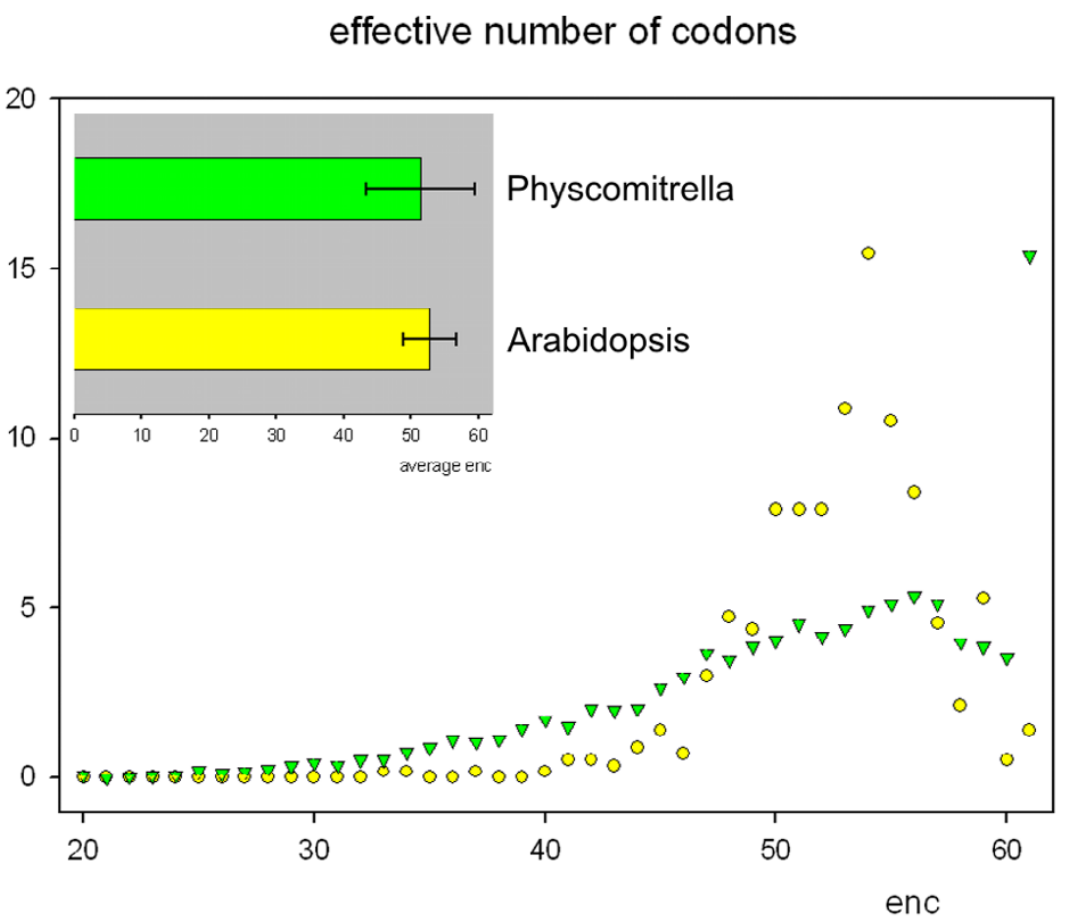

Figure 6

Trinucleotide frequencies and codon usage. a) The averaged Physcomitrella codon fraction usage measured as percentage of the total amount of counted codons is shown as grey diamonds, including a margin of $2 \times$ average absolute deviation (AAD, error bars), in comparison with Arabidopsis (yellow circles). Significantly deviating codons of the sequence subsets are presented as colored circles, namely retained genes (blue), paralogs (red) and orthologs (green). b) The effective number of codons (enc) for Physcomitrella (green) and Arabidopsis (yellow) as a range distribution scatter plot (y axis: \% of analysed genes) and as averaged values (horizontal bar chart; error bars: standard deviation). 
Table 3: Codon usage of Physcomitrella retained genes, orthologs and paralogs

\begin{tabular}{|c|c|c|c|c|c|c|c|c|c|}
\hline sequence set & \# bases & $\begin{array}{c}\text { mean \# of } \\
\text { each triplet }\end{array}$ & G/C content & $\begin{array}{c}\text { \# significant } \\
\text { codon usage } \\
\text { changes }\end{array}$ & $\begin{array}{l}\text { codon usage } \\
\text { towards At }\end{array}$ & $\begin{array}{l}\text { codon usage } \\
\text { away from At }\end{array}$ & $\begin{array}{l}\text { codon over } \\
\text { represented }\end{array}$ & $\begin{array}{l}\text { codon under } \\
\text { represented }\end{array}$ & $\begin{array}{c}\text { significant } \\
\text { changes per } \\
\text { aa }\end{array}$ \\
\hline At mRNAs & $10,755,859$ & 56,020 & 43.32 & n.a. & n.a. & n.a. & n.a. & n.a. & n.a. \\
\hline Pp ORFs & $7,638,122$ & 39,782 & 49.94 & n.a. & n.a. & n.a. & n.a. & n.a. & n.a. \\
\hline retained genes & 77,998 & 406 & 50.30 & 7 & 1 & 6 & 2 & 5 & $\begin{array}{l}\text { Phe under } \\
\text { represented }\end{array}$ \\
\hline paralogs & I,II5,937 & 5,812 & 50.07 & 3 & 1 & 2 & 1 & 2 & none \\
\hline \multirow[t]{2}{*}{ orthologs } & 953,293 & 4,965 & 49.04 & 10 & 8 & 2 & 4 & 6 & $\begin{array}{l}\text { Pro under } \\
\text { reprensented }\end{array}$ \\
\hline & & & & sum & 10 & 10 & 7 & 13 & \\
\hline
\end{tabular}

The predicted Physcomitrella ORF were used as background to check for significant changes in percentage codon fraction usage in the orthologs, paralogs and retained genes (best BLAST hit not among plants). In case of significant deviation (two times average absolute deviation - AAD) from the total set, the direction of the change relative to the Arabidopsis codon usage was checked. Significant deviations are shown enlarged, At = Arabidopsis thaliana, $\mathrm{Pp}=$ Physcomitrella patens.

comitrella (Table 3). It might be argued that the EST-based estimation of G/C content in Physcomitrella is too high because of potential decay of AT-rich sequences [28]. However, when calculating the G/C content for all available 399 full-length CDS from Genbank, the percentage value is also $~ 50 \%(50.67 \%)$. This rate is also found in the retained genes and the Arabidopsis paralogs (Table 3), whereas the ortholog fraction has a significantly lower G/ C content of $\sim 49 \%$, i.e. towards the Arabidopsis nucleotide composition. Codon bias in Physcomitrella is positively correlated with gene expression level and G/C content of the CDS [29]. It was argued that weak natural selection for translational efficiency is the driving force behind codon bias in the moss rather than mutational bias. Given the G/ $\mathrm{C}$ rate of $50 \%$ in the CDS, a mutational bias indeed seems unlikely.

In retained genes, Phenylalanine codons are underrepresented, in the orthologs this is the case for Proline codons. As can also be seen from the G/C content drift mentioned above, the majority of deviating codons in the orthologs changed in the direction of the Arabidopsis percentage usage. For retained genes, it is the direct opposite: the significantly deviating codons in these genes point away from the Arabidopsis codon fraction usage. Orthologs are thought to be functionally equivalent across taxonomic groups. The common ancestor of land plants might have had a G/C content similar to mosses, i.e. around 50\%. In order to preserve efficient functioning of orthologs it might have been necessary to evolve a slightly different codon usage for these genes in mosses, as is e.g. the case in Arabidopsis. The retained genes, on the other hand, are not found in seed plants and do not reflect the codon usage found there.

The average number of synonymous codons that is used in Physcomitrella and Arabidopsis CDS is not significantly different (Fig. 6b, bar chart). However, the percentage distribution of synonymous codon usage, as measured by the effective number of codons (enc), is surprisingly dissimilar (Fig. 6b, scatter plot). Most Arabidopsis coding sequences use a lot of synonymous codons (enc 45-59), whereas Physcomitrella displays a linear percentage increase from low to high values. Interestingly, around $15 \%$ of the moss genes contain no codon bias at all (enc $=61$ ).

\section{Conclusion}

The genome of the ancient land plant Physcomitrella patens, a moss, harbours genes of which at least 30\% have a detectable homolog in seed plants. EST clustering yielded a database that covers a large proportion of the transcriptome, approximately $8 \%$ of the virtual transcripts contain full-length CDS.

Transcripts that are clear homologs of Arabidopsis genes were mapped against the Arabidopsis chromosomes, along with the set of paralogs and orthologs between the two organisms. All three sequence sets could be mapped evenly across the chromosomes, revealing neither hot nor cold spots (despite centromeric regions) nor differences in gene density.

While moss genes resemble those of Arabidopsis, there are significant differences. Introns are larger than those of the seed plant and are also longer than exons within moss, which is not the case in Arabidopsis. The G/C content of exons equals the A/T content. This might reflect a certain tenacity of the mosses' genetic system and its slow mutational rate. These might be necessary characteristics, as due to haploidy in the dominant gametophyte, the chance for the propagation of a disadvantageous change is higher than in a polyploid organism.

Whereas orthologs display a codon fraction usage drift towards Arabidopsis, the contrary is the case for retained 
genes. Thus, evolution of codon usage seems to be correlated with evolutionary history of protein genes. Mutational bias does not seem to play a role in the evolution of moss coding sequences. While the majority of Physcomitrella CDS displays codon bias, there is a significant fraction ( $15 \%)$ of genes that is not biased at all, possibly representing a more ancient nucleotide composition than oberved in Arabidopsis. Splice sites in the moss resemble those in Arabidopsis, however, species-specific prediction models, like the one presented here, are necessary in order to avoid false positives. The same is true for the prediction of ORF based on EST data.

A high proportion of the sequence space of unicellular eukaryotes is covered by moss homologs, which apparently have not been lost since the days of the last common ancestor. The majority of moss genes find their best scoring homolog in plants. However, there are 134 putative retained genes that have their best BLAST hit among other taxonomic groups. Of those, 57 genes are specific to a single taxonomic group, putative functional annotation could be carried out for 25 of these proteins. The functional annotation revealed deviations in the taxonomic distributions: certain sets of genes seem to be shared with specific taxonomic groups, for example, transport proteins with fungi or signal transduction genes with bacteria and animals. Of special interest are genes that are possibly involved in cytotoxicity, metabolism and nucleic acid repair. These genes might be the reasons for some of the extraordinary capabilities of mosses, namely resistance against microbial pathogens, additional secondary pathways (as compared with seed plants) and a high rate of homologous recombination.

\section{Methods \\ Clustering of EST data}

All publicly available protein encoding DNA sequences of Physcomitrella were retrieved using Entrez [30] and divided into 399 "seeds" (full length mRNA sequences) as well as 102,535 EST and other sequences. This dataset is called the Physcomitrella patens public set, or PPP.

A set of 17 moss-specific repetitive elements, detected mainly in the untranslated regions of Physcomitrella genes [31] and used for filtering (see below) is available via cosmoss.org [32]. Filtering, clustering and assembly of EST data were done using the Paracel transcript assembler, PTA [33]. A species-specific parameter set has been developed and is available upon request.

For sequences where electropherograms were available, base-calling was carried out using phred [34]. Base quality values of EST sequences without available sequencer raw data was set arbitrarily to a low value, $10 \%$, and in the case of seed sequences to a higher confidence value of $50 \%$.
Filtering included steps for removal of synthetic (vector/ linker) and low quality sequences as well as of contaminants (homologs of E. coli as well as Physcomitrella mitochondrial, rRNA and chloroplast genes). Low-complexity regions were annotated together with poly-A tails, untranslated regions (UTR, UTRdb see [35]) and repetitive elements (repeats, repbase see [36]), in order not to disturb clustering and assembly. In a final step, sequences containing less than 150 bases of sense characters were removed. For PPP, a total of 100,079 sequences went into the clustering.

Prior to clustering, homologs of the seed sequences were pulled out of the sequence pool and assembled independently. Where possible, sequences were placed into $5^{\prime}$ and 3 ' partitions based on detected poly-A tails and inherent annotated information. Both during clustering and assembly, putative chimeras (cloning artefacts) were detected and tagged. During assembly, contigs were built within clusters and putative splice variants detected. After clustering and assembly, the PPP set contained a total of 26,131 sequences. By using only the longest sequence in each cluster, a non-redundant set of 22,218 sequences was produced. The PP dataset contained 63,685 sequences in the complete and 48,961 sequences in the non-redundant set.

\section{Splice site prediction}

For the splice site prediction, all publicly available pairs of genomic and cDNA/mRNA sequences were retrieved (40 genes). Together with 29 unpublished sequences, these sequences were aligned using MGAlign 1.3.6 [37] in order to determine the splice sites. The procedure yielded a total of 438 exons and 368 introns. The complete dataset is available via cosmoss.org [32].

The sequence logos (Fig. 5a) were created via the web interface at [38] using 10 nucleotides up- and downstream of the donor / acceptor sites.

Suppor vector machine: The software used for training and classification was SVMlight [39], libsvm [40] and svmsplice [41]. The complete set of splice sites was divided into training/testing sets of sizes $10-90 \%$, for each set three samples were drawn. The set containing $90 \%$ of the sites for training proved to yield the best results. Optimization of parameters was done by 10 -fold cross-validation, plotting precision vs. recall and chosing the best curve. The best performing model could be constructed using 50 nucleotides up- and downstream of the splice sites as context with the basepairing feature set of svmsplice and a polynomial kernel function of $4^{\text {th }}$ order. 


\section{BLAST searches and filtering}

BLAST searches were carried out using Paracel BLAST [33], a parallelized version of BLAST 2 [42], on amino acid level whenever applicable. In order to exclude random hits which are not based on true sequence homology, alignments had to contain at least 30\% identical positions and a minimum length of 100 amino acid characters. This rigorous filtering excludes some true positive hits but removes almost all false positives [43]. Putative fulllength CDS had to pass the same filtering. In addition, in this case only those hits were counted that covered at least $90 \%$ of the subjects length. For the determination of identical sequences, BLASTN was performed and hits were filtered to be at least 95\% identical and 300 nucleotides long. Non-redundant hits were counted by removing all subjects that were present more than once in the search result.

\section{Additional sequence datasets}

The predicted coding sequences of rice $(56,056$ sequences) and Arabidopsis (28,581 sequences) genes were taken from release 1.0 and 4.0 of the TIGR database [44], respectively. The taxprot dataset $(3,346,100$ sequences, see Table 1 for details) was created by downloading the respective sequences from Genbank [30] using appropriate Entrez queries. All three datasets consist of amino acid sequences. The Arabidopsis thaliana chromosome sequences were retrieved from Genbank [45].

\section{ORF prediction}

ESTScan 2.0 [46] was used to predict open reading frames. The species-specific model for Physcomitrella was built by using the 399 public full length seed sequences (complete mRNAs) mentioned above. ORF were predicted from the clustered EST data (non-redundant datasets). For the PPP set, 19,081 ORF were predicted; 34,981 for the PP set. Predictions were done using both the Arabidopsis and the Physcomitrella model for comparison. Manual inspection of several known CDS revealed that the Arabidopsis-based prediction contained false-positive stretches, which was not the case for the Physcomitrella-based prediction. Although the Physcomitrella model predicted a lower number of ORF, it was used in order to keep false-positives to a minimum.

\section{Codon usage}

Four different sets of coding sequences were used (see table 3). A set of 7,765 well annotated Arabidopsis mRNAs was retrieved using Entrez. The Physcomitrella datasets contained the predicted ORF for the complete PPP set $(19,081$ sequences), the Arabidopsis paralogs $(1,659)$ and orthologs $(1,476)$ described above as well as the putatively retained genes not found in higher plants (134). The smallest set contained 77,998 nucleotides and thus a theoretical average of 406 instances of each triplet, which allows significant analyses.

Nucleotide frequencies were calculated with the GCG 10.3 [47] software composition. Codon usage fractions for individual datasets were calculated as percentage of the respective total amount of counted codons. Absolute deviations in comparison to the full Physcomitrella ORF set were calculated for the three subsets (retained genes, Arabidopsis orthologs and paralogs). The computed mean value over all sets (average absolute deviation) was 0.069 . Codon fraction usage deviation was counted as significant only if it differed at least twice as much $(+/-0.138 \%)$ from the full set.

The effective number of codons (enc) was calculated using CodonW (J. Peden, [48]). The enc values range from 20 (maximum bias, i.e. only one synonymous codon is used per amino acid) to 61 (no bias, all synonymous codons are being used).

\section{Abbreviations}

$\mathrm{CDS}=$ coding sequence $(\mathrm{s}), \mathrm{EST}=$ expressed sequence $\operatorname{tag}(\mathrm{s}), \mathrm{ORF}=$ open reading frame $(\mathrm{s})$

\section{Authors' contributions}

SAR carried out most of the analyses, drafted the manuscript and designed the work. DF carried out the splice site prediction. DL participated in the analyses and generated the databases and the web interface. RR participated in drafting and conception of the manuscript. All authors read and approved the final manuscript.

\section{Acknowledgements}

We would like to thank Sven Degroeve (University of Gent, Belgium) for providing of and assistance with svmsplice as well as Hans Stenøien, Colette Matthewman and several anonymous reviewers for helpful comments on the manuscript.

\section{References}

I. Theissen G, Münster T, Henschel K: Why don't mosses flower? New Phytologist 200I, 150: I-8.

2. Miller ND: Tertiary and quarternary fossils. In New manual of Bryology Volume 2. Edited by: Schuster RM. Miyazaki: Hattori Bot Lab; 1984:11 194-1232.

3. Frahm J-P: Moose - lebende Fossilien. BuZ I994, 24(3): I20-I 24.

4. Chiang TY, Schaal BA: Molecular evolution and phylogeny of the atpB-rbcL spacer of chloroplast DNA in the true mosses. Genome 2000, 43(3):4I7-426.

5. Hohe A, Egener T, Lucht JM, Holtorf H, Reinhard C, Schween G, Reski R: An improved and highly standardised transformation procedure allows efficient production of single and multiple targeted gene-knockouts in a moss, Physcomitrella patens. Curr Genet 2004, 44(6):339-347.

6. Reski R, Cove DJ: Quick guide: Physcomitrella patens. Curr Biology 2004, I4:R26I-R262.

7. Schaefer DG: A New Moss Genetics: Targeted Mutagenesis in Physcomitrella patens. Annual Review of Plant Physiology 2002, 53:477-50I.

8. Nishiyama T, Fujita T, Shin IT, Seki M, Nishide H, Uchiyama I, Kamiya A, Carninci P, Hayashizaki Y, Shinozaki K, et al: Comparative genomics of Physcomitrella patens gametophytic transcrip- 
tome and Arabidopsis thaliana: implication for land plant evolution. Proceedings of the National Academy of Sciences of the United States of America 2003, I 00( I 3):8007-80 I 2

9. Rensing SA, Rombauts S, Van de Peer Y, Reski R: Moss transcriptome and beyond. Trends in Plant Science 2002, 7( I 2):535-538.

10. Frank W, Ratnadewi D, Reski R: Physcomitrella patens is highly tolerant against drought, salt and osmotic stress. Planta 2005 , 220:384-394.

II. Kroemer K, Reski R, Frank W: Abiotic stress response in the moss Physcomitrella patens: evidence for an evolutionary alteration in signaling pathways in land plants. Plant Cell Reports 2004, 22( I I):864-870.

12. Richter U, Kiessling J, Hedtke B, Decker E, Reski R, Borner T, Weihe A: Two RpoT genes of Physcomitrella patens encode phagetype RNA polymerases with dual targeting to mitochondria and plastids. Gene 2002, 290(I-2):95-105.

13. Kiessling J, Martin A, Gremillon L, Rensing SA, Nick P, Sarnighausen E, Decker EL, Reski R: Dual targeting of plastid division protein FtsZ to chloroplasts and the cytoplasm. EMBO Rep 2004, 5(9):889-894.

14. Girke T, Schmidt H, Zahringer U, Reski R, Heinz E: Identification of a novel delta 6-acyl-group desaturase by targeted gene disruption in Physcomitrella patens. The Plant Journal 1998, I 5(I):39-48

15. Koprivova A, Meyer AJ, Schween G, Herschbach C, Reski R, Kopriva S: Functional knockout of the adenosine 5'-phosphosulfate reductase gene in Physcomitrella patens revives an old route of sulfate assimilation. Journal of Biological Chemistry 2002, 277(35):32195-3220I.

16. Zank TK, Zahringer U, Beckmann C, Pohnert G, Boland W, Holtorf $H$, Reski R, Lerchl J, Heinz E: Cloning and functional characterisation of an enzyme involved in the elongation of Delta6-polyunsaturated fatty acids from the moss Physcomitrella patens. The Plant Journal 2002, 3 I(3):255-268.

17. Mushegian AR, Garey JR, Martin J, Liu LX: Large-scale taxonomic profiling of eukaryotic model organisms: a comparison of orthologous proteins encoded by the human, fly, nematode, and yeast genomes. Genome Research 1998, 8(6):590-598.

18. Remm M, Storm CE, Sonnhammer EL: Automatic clustering of orthologs and in-paralogs from pairwise species comparisons. J Mol Biol 200I, 3 I4(5): I04I-I 052.

19. Brun F, Gonneau M, Doutriaux MP, Laloue M, Nogue F: Cloning of the PpMSH-2 cDNA of Physcomitrella patens, a moss in which gene targeting by homologous recombination occurs at high frequency. Biochimie 2001, 83(I I-1 2):1003-1008.

20. Mikami K, Repp A, Graebe-Abts E, Hartmann E: Isolation of cDNAs encoding typical and novel types of phosphoinositide-specific phospholipase $\mathbf{C}$ from the moss Physcomitrella patens. Journal of Experimental Botany 2004, 55(40I): I 437-I439.

21. Takezawa D, Minami A: Calmodulin-binding proteins in bryophytes: identification of abscisic acid-, cold-, and osmotic stress-induced genes encoding novel membrane-bound transporter-like proteins. Biochemical and Biophysical Research Communications 2004, 3 I 7(2):428-436.

22. Von Schwartzenberg K, Schultze W, Kassner H: The moss Physcomitrella patens releases a tetracyclic diterpene. Plant Cell Reports 2004, 22(I 0):780-786.

23. Strepp R, Scholz S, Kruse S, Speth V, Reski R: Plant nuclear gene knockout reveals a role in plastid division for the homolog of the bacterial cell division protein FtsZ, an ancestral tubulin. Proceedings of the National Academy of Sciences of the United States of America 1998, 95(8):4368-4373.

24. Hohe A, Reski R: A tool for understanding homologous recombination in plants. Plant Cell Reports 2003, 2 I ( I 2): I I35- I I 42.

25. Hebsgaard SM, Korning PG, Tolstrup N, Engelbrecht J, Rouze P, Brunak S: Splice site prediction in Arabidopsis thaliana pre-mRNA by combining local and global sequence information. Nucleic Acids Research 1996, 24(I 7):3439-3452.

26. Deutsch M, Long M: Intron-exon structures of eukaryotic model organisms. Nucleic Acids Research 1999, 27( I 5):3219-3228.

27. Rensing SA, Kiessling J, Reski R, Decker EL: Diversification of ftsZ during early land plant evolution. J Mol Evol 2004, 58(2): I 54- I62.

28. Margulies EH, Kardia SL, Innis JW: Identification and prevention of a GC content bias in SAGE libraries. Nucleic Acids Research 200I, 29( I 2):E60-60.
29. Stenoien HK: Adaptive basis of codon usage in the haploid moss Physcomitrella patens. Heredity 2005, 94:87-93.

30. NCBI Entrez [http://www.ncbi.nlm.nih.gov/Entrez]

31. Rensing SA, Lang D, Reski R: In silico prediction of UTR repeats using clustered EST data. In Proceedings of the German Conference on Bioinformatics: 2003 Munich, Germany: Belleville Verlag Michael Farin; 2003: II7-122.

32. cosmoss.org [http://www.cosmoss.org]

33. Paracel [http://www.paracel.com]

34. Ewing B, Hillier L, Wendl MC, Green P: Base-calling of automated sequencer traces using phred. I. Accuracy assessment. Genome Res 1998, 8(3): 175-185

35. Pesole G, Grillo G, Liuni S: Databases of mRNA untranslated regions for metazoa. Computers \& Chemistry 1996, 20(I): |4|- | 44.

36. Jurka J: Repbase update: a database and an electronic journal of repetitive elements. Trends in Genetics 2000, I6(9):418-420.

37. Lee BT, Tan TW, Ranganathan S: MGAlignlt: A web service for the alignment of $m R N A / E S T$ and genomic sequences. Nucleic Acids Research 2003, 3 I ( I 3):3533-3536.

38. Sequence logo [http://www.cbs.dtu.dk/ gorodkin/appl/slogo.html]

39. SVMlight [http://svmlight.joachims.org]

40. libsvm [http://www.csie.ntu.edu.tw/ cjlin/libsvm]

41. Degroeve S, De Baets B, Van De Peer Y, Rouze P: Feature subset selection for splice site prediction. Bioinformatics 2002, I 8(2):S75-S83.

42. Altschul SF, Madden TL, Schaffer AA, Zhang J, Zhang Z, Miller W, Lipman DJ: Gapped BLAST and PSI-BLAST: a new generation of protein database search programs. Nucleic Acids Research 1997, 25(I 7):3389-3402.

43. Rost B: Twilight zone of protein sequence alignments. Protein Eng 1999, I 2(2):85-94.

44. TIGR [http://www.tigr.org]

45. Genbank eukaryotic genomes [http://www.ncbi.nlm.nih.gov/ genomes/static/euk g.html]

46. Iseli C, Jongeneel CV, Bucher P: ESTScan: a program for detecting, evaluating, and reconstructing potential coding regions in EST sequences. International Conference on Intelligent Systems for Molecular Biology: 1999 1999: I38-148.

47. Accelrys [http://www.accelrys.com]

48. CodonW [http://www.molbiol.ox.ac.uk/cu/]



